\title{
On solutions of certain limit boundary value problems
}

Cite as: AIP Conference Proceedings 2293, 110006 (2020); https://doi.org/10.1063/5.0026562

Published Online: 25 November 2020

\section{Shaban Kutaiba, and Vladimir Vasilyev}
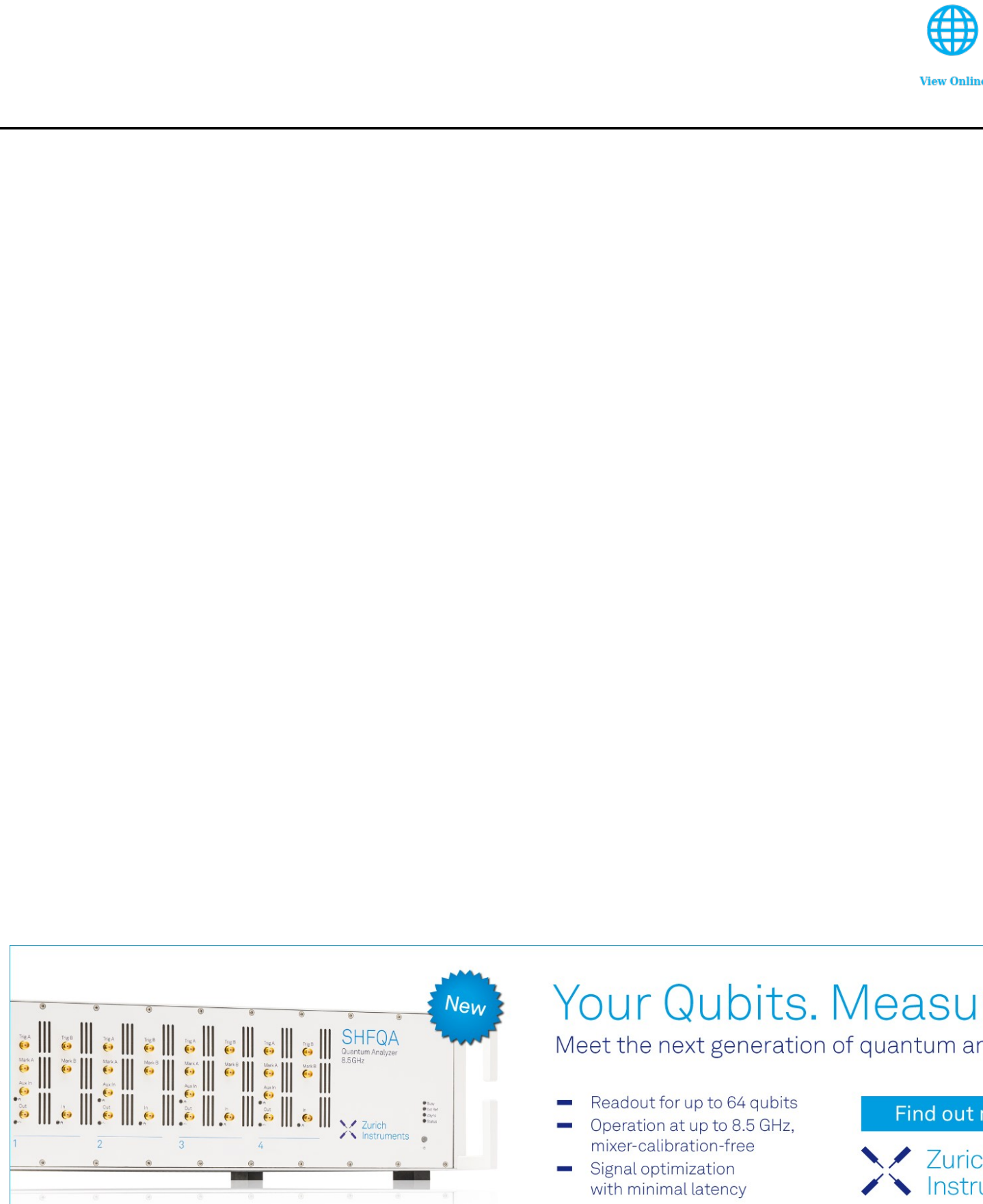

\section{Your Qubits. Measured.}

Meet the next generation of quantum analyzers

- Readout for up to 64 qubits

- Operation at up to $8.5 \mathrm{GHz}$,

mixer-calibration-free

- Signal optimization with minimal latency 


\title{
On Solutions of Certain Limit Boundary Value Problems
}

\author{
Shaban Kutaiba ${ }^{1, \mathrm{~b}}$ and Vladimir Vasilyev ${ }^{1, \mathrm{a}}$ \\ ${ }^{1}$ Chair of Differential Equations, Belgorod State National Research University, Pobedy street 85, Belgorod 308015, \\ Russia \\ a) Corresponding author: vbv57@inbox.ru \\ b)1164572@bsu.edu.ru
}

Abstract. We study special boundary value problem in a plane sector for a model elliptic pseudo-differential equation in SobolevSlobodetskii spaces. Using special factorization for an elliptic symbol we construct the solutions and study the case when size of the cone tends to zero. It was shown that such a limit can exist if the boundary function is solution of certain integral equation.

\section{Introduction}

Earlier one of the authors studied elliptic pseudo-differential equations in domains with singular points at a boundary [1]. The following equation

$$
(A u)(x)=v(x), \quad x \in C,
$$

was studied, where $C$ is a convex cone in Euclidean space $\mathbb{R}^{m}, A$ is a pseudo-differential operator with the symbol $A(\xi)$ satisfying the condition

$$
c_{1}(1+|\xi|)^{\alpha} \leq|A(\xi)| \leq c_{2}(1+|\xi|)^{\alpha}
$$

and it looks as follows

$$
(A u)(x)=\int_{C} \int_{\mathbb{R}^{m}} A(\xi) e^{i(x-y) \cdot \xi} u(y) d \xi d y, \quad x \in C .
$$

The main goal is obtaining conditions for unique solvability for the equation (1) in appropriate functional spaces, or invertibility conditions for the operator $A$. To describe such conditions a concept of the wave factorization for an elliptic symbol was introduced [1]. Unfortunately, a number of solutions depends on an index of the wave factorization [1] and to extract the unique solution one needs some additional conditions.

Similar problems were considered from different sides in papers $[2,3,4,5]$. Some results were devoted to very special case in which size of a cone tends to zero $[4,5]$. There are certain interesting results concerning such cones. Here we consider simple plane case when one needs to add some condition to the equation to obtain the unique solution.

We use usual Sobolev-Slobodetskii spaces $H^{s}\left(\mathbb{R}^{m}\right)[6,1]$ with the norm

$$
\|u\|_{s}^{2}=\int_{\mathbb{R}^{m}}|\tilde{u}(\xi)|^{2}(1+|\xi|)^{2 s} d \xi
$$

and $\sim$ over a function denotes its Fourier transform

$$
\tilde{u}(\xi)=\int_{\mathbb{R}^{m}} e^{i x \cdot \xi} u(x) d x .
$$




\section{A general solution}

Here we will study 2-dimensional case and the cone $C_{+}^{a}=\left\{x \in \mathbb{R}^{2}: x_{2}>a\left|x_{1}\right|, a>0\right\}$ under the condition $1 / 2<$ $\mathfrak{x}-s<3 / 2$, where $æ$ is the index of wave factorization (see below), $s$ is an exponent of Sobolev-Slobodetskii space $H^{s}\left(C_{+}^{a}\right)$. The latter space consists of functions from $H^{s}\left(\mathbb{R}^{m}\right)$ with support in $\overline{C_{+}^{a}}$.

For simplicity we put $v \equiv 0$. Let $C_{+}^{a}$ be a conjugate cone for the cone $C_{+}^{a}$ :

$$
\stackrel{*}{a}_{+}^{a}=\left\{x \in \mathbb{R}^{2}: x=\left(x_{1}, x_{2}\right), a x_{2}>\left|x_{1}\right|\right\},
$$

$C_{-}^{a} \equiv-C_{+}^{a}, T\left(C_{+}^{a}\right)$ be a radial tube domain over the cone $C_{+}^{a}$, i. e. a domain of a two-dimensional complex space $\mathbb{C}^{2}$ of the following type $\mathbb{R}^{2}+i C_{+}^{a}$.

We remind here that the wave factorization of an elliptic symbol $A(\xi)$ is called its representation in the form [1]

$$
A(\xi)=A_{\neq}(\xi) A_{=}(\xi),
$$

where factors $A_{\neq}(\xi), A_{=}(\xi)$ must satisfy the following conditions:

1) $A_{\neq}(\xi), A_{=}(\xi)$ are defined for all $\xi \in \mathbb{R}^{2}$ may be except $\left\{\xi \in \mathbb{R}^{2}:\left|\xi_{1}\right|^{2}=a^{2} \xi_{2}^{2}\right\}$;

2) $A_{\neq}(\xi), A_{=}(\xi)$ admit an analytic continuation into radial tube domains

$T\left(\stackrel{*}{C_{+}^{a}}\right), T\left({\stackrel{*}{C_{-}^{a}}}^{a}\right)$ respectively with estimates

$$
\begin{gathered}
\left|A_{\neq}^{ \pm 1}(\xi+i \tau)\right| \leq c_{1}(1+|\xi|+|\tau|)^{ \pm \mathfrak{x}}, \\
\left|A_{=}^{ \pm 1}(\xi-i \tau)\right| \leq c_{2}(1+|\xi|+|\tau|)^{ \pm(\alpha-\mathfrak{x})}, \forall \tau \in C_{+}^{*} .
\end{gathered}
$$

The number $æ \in \mathbb{R}$ is called an index of the wave factorization.

If the symbol $A(\xi)$ admits the wave factorization [1] then one can show [2] that a general solution of the equation (1) in Sobolev-Slobodetskii space $H^{s}\left(C_{+}^{a}\right)$ in Fourier image has the following form

$$
\begin{gathered}
\tilde{u}(\xi)=\frac{\tilde{c}_{0}\left(\xi_{1}+a \xi_{2}\right)+\tilde{c}_{0}\left(\xi_{1}-a \xi_{2}\right)}{2 A_{\neq}\left(\xi_{1}, \xi_{2}\right)}+ \\
+A_{\neq}^{-1}\left(\xi_{1}, \xi_{2}\right)\left(v \cdot p \cdot \frac{i}{2 \pi} \int_{-\infty}^{+\infty} \frac{\tilde{c}_{0}(\eta) d \eta}{\xi_{1}+a \xi_{2}-\eta}-v \cdot p \cdot \frac{i}{2 \pi} \int_{-\infty}^{+\infty} \frac{\tilde{c}_{0}(\eta) d \eta}{\xi_{1}-a \xi_{2}-\eta}\right),
\end{gathered}
$$

where $c_{0}$ is an arbitrary function from $H^{s-\mathfrak{x}+1 / 2}(\mathbb{R})$.

Let us denote

$$
v \cdot p \cdot \frac{i}{\pi} \int_{-\infty}^{+\infty} \frac{\tilde{c}_{0}(\eta) d \eta}{\xi_{1}+a \xi_{2}-\eta} \equiv \tilde{d}_{0}\left(\xi_{1}+a \xi_{2}\right), \quad v \cdot p \cdot \frac{i}{\pi} \int_{-\infty}^{+\infty} \frac{\tilde{c}_{0}(\eta) d \eta}{\xi_{1}-a \xi_{2}-\eta} \equiv \tilde{d}_{0}\left(\xi_{1}-a \xi_{2}\right) .
$$

Then we have

$$
\begin{gathered}
\tilde{u}\left(\xi_{1}, \xi_{2}\right)=\frac{\tilde{c}_{0}\left(\xi_{1}+a \xi_{2}\right)+\tilde{c}_{0}\left(\xi_{1}-a \xi_{2}\right)+\tilde{d}_{0}\left(\xi_{1}+a \xi_{2}\right)-\tilde{d}_{0}\left(\xi_{1}-a \xi_{2}\right)}{2 A_{\neq}\left(\xi_{1}, \xi_{2}\right)} \equiv \\
\frac{\tilde{c}\left(\xi_{1}+a \xi_{2}\right)+\tilde{d}\left(\xi_{1}-a \xi_{2}\right)}{2 A_{\neq}\left(\xi_{1}, \xi_{2}\right)},
\end{gathered}
$$

where we put $\tilde{c}\left(\xi_{1}+a \xi_{2}\right) \equiv \tilde{c}_{0}\left(\xi_{1}+a \xi_{2}\right)+\tilde{d}_{0}\left(\xi_{1}+a \xi_{2}\right), \tilde{d}\left(\xi_{1}-a \xi_{2}\right) \equiv \tilde{c}_{0}\left(\xi_{1}-a \xi_{2}\right)-\tilde{d}_{0}\left(\xi_{1}-a \xi_{2}\right)$.

Now main problem under consideration what kind of additional conditions we need to have for obtaining the unique solution of the equation (1).

Let us assume that we know the following integral

$$
\int_{-\infty}^{+\infty} u\left(x_{1}, x_{2}\right) d x_{2} \equiv g\left(x_{1}\right)
$$


For the Fourier images it means the following

$$
\tilde{u}\left(\xi_{1}, 0\right)=\tilde{g}(\xi)
$$

and according to the formula (2) we have

$$
\frac{\tilde{c}_{0}\left(\xi_{1}\right)}{A_{\neq}\left(\xi_{1}, 0\right)}=\tilde{g}\left(\xi_{1}\right) .
$$

Thus at least formally we can find the function

$$
\tilde{c}_{0}\left(\xi_{1}\right)=A_{\neq}\left(\xi_{1}, 0\right) \tilde{g}\left(\xi_{1}\right)
$$

and then using formulas (2) we find $\tilde{d}_{0}\left(\xi_{1}\right)$. Hence, the formula (3) gives the solution of the equation (1). Finally the solution of the equation (1) under the condition (4) takes the following form

$$
\begin{gathered}
\tilde{u}\left(\xi_{1}, \xi_{2}\right)=\frac{A_{\neq}\left(\xi_{1}+a \xi_{2}, 0\right) \tilde{g}\left(\xi_{1}+a \xi_{2}\right)+A_{\neq}\left(\xi_{1}-a \xi_{2}, 0\right) \tilde{g}\left(\xi_{1}-a \xi_{2}\right)}{2 A_{\neq}\left(\xi_{1}, \xi_{2}\right)}+ \\
\frac{1}{2 A_{\neq}\left(\xi_{1}, \xi_{2}\right)} v \cdot p \cdot \frac{i}{\pi} \int_{-\infty}^{+\infty} \frac{A_{\neq}(\eta, 0) \tilde{g}(\eta) d \eta}{\xi_{1}+a \xi_{2}-\eta}-\frac{1}{2 A_{\neq}\left(\xi_{1}, \xi_{2}\right)} v \cdot p \cdot \frac{i}{\pi} \int_{-\infty}^{+\infty} \frac{A_{\neq}(\eta, 0) \tilde{g}(\eta) d \eta}{\xi_{1}-a \xi_{2}-\eta}
\end{gathered}
$$

\section{Limit case}

What we can say on $\tilde{u}(\xi)$ if $a \rightarrow \infty$ ? This case corresponds to a thin cone for which its size tends to zero.

We can apply the change of variables

$$
\left\{\begin{array}{l}
t_{1}=\xi_{1}+a \xi_{2} \\
t_{2}=\xi_{1}-a \xi_{2}
\end{array}\right.
$$

and denote

$$
a_{\neq}\left(t_{1}, t_{2}\right) \equiv A_{\neq}\left(\frac{t_{1}+t_{2}}{2}, \frac{t_{1}-t_{2}}{2 a}\right) .
$$

Then we rewrite denoting $\tilde{U}\left(t_{1}, t_{2}\right) \equiv \tilde{u}\left(\frac{t_{1}+t_{2}}{2}, \frac{t_{1}-t_{2}}{2 a}\right)$

$$
\begin{gathered}
\tilde{U}\left(t_{1}, t_{2}\right)=\frac{A_{\neq}\left(t_{1}, 0\right) \tilde{g}\left(t_{1}\right)+A_{\neq}\left(t_{2}, 0\right) \tilde{g}\left(t_{2}\right)}{2 a_{\neq}\left(t_{1}, t_{2}\right)}+ \\
\frac{1}{2 a_{\neq}\left(t_{1}, t_{2}\right)} v \cdot p \cdot \frac{i}{\pi} \int_{-\infty}^{+\infty} \frac{A_{\neq}(\eta, 0) \tilde{g}(\eta) d \eta}{t_{1}-\eta}-\frac{1}{2 a_{\neq}\left(t_{1}, t_{2}\right)} v \cdot p \cdot \frac{i}{\pi} \int_{-\infty}^{+\infty} \frac{A_{\neq}(\eta, 0) \tilde{g}(\eta) d \eta}{t_{2}-\eta}
\end{gathered}
$$

Then we have under $a \rightarrow+\infty$ the following relation

$$
\begin{gathered}
\tilde{u}\left(\frac{t_{1}+t_{2}}{2}, 0\right)=\tilde{U}\left(t_{1}, t_{2}\right)=\frac{A_{\neq}\left(t_{1}, 0\right) \tilde{g}\left(t_{1}\right)+A_{\neq}\left(t_{2}, 0\right) \tilde{g}\left(t_{2}\right)}{2 a_{\neq}\left(t_{1}, t_{2}\right)}+ \\
\frac{1}{2 a\left(t_{1}, t_{2}\right)} v \cdot p \cdot \frac{i}{\pi} \int_{-\infty}^{+\infty} \frac{A_{\neq}(\eta, 0) \tilde{g}(\eta) d \eta}{t_{1}-\eta}-\frac{1}{2 a_{\neq}\left(t_{1}, t_{2}\right)} v \cdot p \cdot \frac{i}{\pi} \int_{-\infty}^{+\infty} \frac{A_{\neq}(\eta, 0) \tilde{g}(\eta) d \eta}{t_{2}-\eta}
\end{gathered}
$$

Let us denote $A_{\neq}(t, 0) \tilde{g}(t) \equiv G(t)$ and $\lim _{a \rightarrow+\infty} a_{\neq}\left(t_{1}, t_{2}\right) \equiv h\left(t_{1}, t_{2}\right)$. Therefore according to the condition (4) we have

$$
2 h\left(t_{1}, t_{2}\right) \tilde{g}\left(\frac{t_{1}+t_{2}}{2}\right)=G\left(t_{1}\right)+G\left(t_{2}\right)+(S G)\left(t_{1}\right)-(S G)\left(t_{2}\right)
$$

where

$$
(S G)(t)=v \cdot p \cdot \frac{i}{\pi} \int_{-\infty}^{+\infty} \frac{G(\eta) d \eta}{t-\eta}
$$

Taking into account our above considerations we obtain the following result.

Theorem. If the symbol $A\left(\xi_{1}, \xi_{2}\right)$ admits the wave factorization with respect to $C_{+}^{a}$ for enough large a then under $a \rightarrow+\infty$ the limit (5) exists, boundary value problem (1),(4) is solvable iff the condition (6) holds. 


\section{Conclusion}

Here some plane problems were considered. The same approach can be realized in a multidimensional space also. Such situations will be studied in our forthcoming papers.

\section{Acknowledgement}

This work was supported by the State contract of the Russian Ministry of Education and Science (contract No 1.7311.2017/8.9).

\section{REFERENCES}

[1] V. B. Vasil'ev, Wave Factorization of Elliptic Symbols: Theory and Applications. Introduction to the Theory of Boundary Value Problems in Non-Smooth Domains (Kluwer Academic Publishers, Dordrecht-BostonLondon, 2000), pp. 1-172.

[2] V. B. Vasilyev, Opusc. Math. 39, 109-124 (2019).

[3] V. B. Vasilyev, Math. Meth. Appl. Sci. 41, 9252-9263 (2018).

[4] V. B. Vasilyev, "Asymptotical analysis of singularities for pseudo differential equations in canonical nonsmooth domainsr," in Integral Methods in Science and Engineering. Computational and Analytic Aspects, edited by C. Constanda and P. J. Harris (Birkhauser, Boston, 2011), pp. 379-390.

[5] V. B. Vasilyev, Bound. Value Probl. 116, 1-13 (2017).

[6] G. Eskin, Boundary Value Problems for Elliptic Pseudodifferential Equations (AMS, Providence (RI), 1981), pp. 1-375. 